\title{
Genome-wide identification of cancer/testis genes and their association with prognosis in a pan-cancer analysis
}

\author{
Vandeclecio Lira da Silva ${ }^{1,2,3, *}$, André Faustino Fonseca ${ }^{1,2,3, *}$, Marbella Fonseca ${ }^{1}$, \\ Thayna Emilia da Silva ${ }^{1}$, Ana Carolina Coelho ${ }^{1,3}$, José Eduardo Kroll ${ }^{1,3,4}$, Jorge \\ Estefano Santana de Souza ${ }^{3,5}$, Beatriz Stransky ${ }^{3,6}$ Gustavo Antonio de Souza ${ }^{1,3}$ and \\ Sandro José de Souza1,3 \\ ${ }^{1}$ Instituto do Cérebro, UFRN, Natal, Brazil \\ ${ }^{2}$ Ph.D. Program in Bioinformatics, UFRN, Natal, Brazil \\ ${ }^{3}$ Bioinformatics Multidisciplinary Environment (BioME), Digital Metropolis Institute, UFRN, Natal, Brazil \\ ${ }^{4}$ Instituto de Bioinformática e Biotecnologia, Natal, Brazil \\ ${ }^{5}$ Instituto Metrópole Digital, UFRN, Natal, Brazil \\ ${ }^{6}$ Departmento de Engenharia Biomédica, UFRN, Natal, Brazil \\ *These authors have contributed equally to this work \\ Correspondence to: Sandro José de Souza, email: sandro@neuro.ufrn.br \\ Keywords: cancer/testis, cancer antigens, biomarkers, prognosis \\ Received: June 03, $2017 \quad$ Accepted: August 17, $2017 \quad$ Published: October 10, 2017 \\ Copyright: Silva et al. This is an open-access article distributed under the terms of the Creative Commons Attribution License 3.0 \\ (CC BY 3.0), which permits unrestricted use, distribution, and reproduction in any medium, provided the original author and source \\ are credited.
}

\section{ABSTRACT}

Cancer/testis (CT) genes are excellent candidates for cancer immunotherapies because of their restrict expression in normal tissues and the capacity to elicit an immune response when expressed in tumor cells. In this study, we provide a genomewide screen for CT genes with the identification of 745 putative CT genes. Comparison with a set of known CT genes shows that 201 new CT genes were identified. Integration of gene expression and clinical data led us to identify dozens of CT genes associated with either good or poor prognosis. For the CT genes related to good prognosis, we show that there is a direct relationship between CT gene expression and a signal for CD8+ cells infiltration for some tumor types, especially melanoma.

\section{INTRODUCTION}

Genes with a restricted pattern of expression in normal tissues and expression in tumors cells are excellent candidates for biomarkers and therapeutic targets. Among these genes, cancer/testis (CT) genes are the most promising with several clinical trials under way [1]. These $\mathrm{CT}$ genes are exclusive or predominantly expressed in testis among normal tissues but are also expressed in a variety of tumor types [2]. Few authors have suggested that these genes may be considered as cancer-germline (CG) genes, as they can also present regular expression in ovary and placenta [3,4]. When protein products of $\mathrm{CT}$ genes elicit an immune response, they are called CT antigens [3]. Methodologies, like SEREX and protein arrays, have identified several CT genes as $\mathrm{CT}$ antigens.
Cellular and humoral immune responses have been observed for many $\mathrm{CT}$ antigens in patients bearing a variety of tumor types [5-7], making them good candidate targets for cancer immunotherapy, like cancer vaccination, adoptive T-cell transfer with chimeric T-cell receptors or in combination with conventional cytotoxic therapies [8-12].

Although there is a lack of consensual classification of $\mathrm{CT}$ genes, authors have divided them into two distinct groups [13]. The first one is organized in multigene families generally located on the $\mathrm{X}$ chromosome, where they comprise approximately $10 \%$ of the genes on that chromosome (CT-X genes) and show heterogeneous expression in cancer tissues that increase during tumor evolution and can elicit immune responses in cancer patients. The other group comprises the non-X CT genes since they are located on autosomal chromosomes. This last class does not appear to exist as 
multigene families and are often expressed during meiosis [14]. In 2009, data from about 70 families of cancer testis antigen, with more than 200 members, were gathered into a database (http://www.cta.lncc.br/). This CT Database provides information about $\mathrm{CT}$ genes including names and aliases, genomic location among others, but has not incorporated data from new technologies and contains less than $250 \mathrm{CT}$ genes [15]. Moreover, several other genomewide analyses have been reported. Hofmann et al. [14], for example, used a combination of CAGE, MPSS and RT-PCR to perform a survey of cancer/testis genes. They identified more than 30 candidate CT genes. More recently, our group characterized the human surfaceome and identified 14 putative CT genes coding for cell surface proteins [16]. Using public microarray data, two genes FMR1NB and TMEM31 were characterized as CT genes coding for cell surface proteins, which render them excellent candidates for targeted therapies [16]. One of them, FMR1NB, was found to elicit immune response in sarcoma patients [17]. While the project reported here was underway, Wang et al. [5] reported a systematic identification of CT genes in 19 tumor types.

The development of next-generation sequencing (NGS) technologies catalyzed a series of projects whose primary objective was to genetically characterize a cohort of cancer patients and associate that information with clinical data. The most successful of these projects was "The Cancer Genome Atlas" (TCGA) that integrated information for more than 11,000 patients from a variety of tumor types. NGS technologies have also allowed a better characterization of the human transcriptome derived from healthy cells and tissues. Projects like the Human Body Map (HBM) (GEO accession: GSE30611), the Genotype-Tissue Expression (GTEx) [18] and the Human Protein Atlas (HPA) [19] have deep sequenced the human transcriptome of dozens of cell types.

While the genome-wide screens performed so far were necessary for a better characterization of the universe of CT genes, most of them were executed at a time in which these NGS technologies were not widely available. Yao [20] have recently used TCGA data to explore CT genes. They, however, restricted their analysis to a subset of known CT genes. On the other hand, Wang et al. [5] used TCGA data to perform a genome-wide screen for CT genes. In the present paper, we have integrated information derived from both HBM/GTEx/HPA and TCGA to provide a complete genome survey of CT genes with the characterization of 201 new putative CT genes. By using mass-spectrometry data, it is shown that several of our putative CT genes exist at the protein level in some tumor types. Finally, CT genes associated with either a good or poor outcome are identified. For some CT genes associated to good prognosis, an association to CD8+ cells infiltration is observed. We propose that CT genes whose expression is related to a good outcome are excellent candidates for immunotherapeutic approaches.

\section{RESULTS}

\section{Identification of genes predominantly (or exclusively) expressed in testis}

Assuming that CT genes are predominant or exclusively expressed in testis when compared to the remaining tissues, we used RNA-Seq data from three different sources to identify genes with expression bias to the testis. Absolute transcript level in each tissue was converted to a proportional score (transcript level in a tissue divided by the sum of levels in all tissues), and a threshold of at least 0.9 was used to select genes preferentially or exclusively expressed in testis. A threshold of 0.9 would imply that at least $90 \%$ of all expression of that gene in all analyzed tissues was derived from testis. HBM (GEO accession: GSE30611), GTEx [18] and the HPA [19] datasets, all reporting RNASeq data from a variety of normal tissues, were used to select genes preferentially (or exclusively) expressed in testis. The resulting selection, detailed in Figure 1A, contained a set of 1,103 non-redundant genes based on the three sources used in our analysis (Figure 1B and Supplementary Table 1 for a complete list of testis-biased genes). A gene ontology analysis in this gene set showed, as expected, a strong bias towards biological processes related to germline cells (Figure 1C).

To make available a set of putative CT genes identified using more stringent criteria, we modified our pipeline to select genes with a proportion score of at least 0.99 . This selection generated a list of 793 genes with an exclusive expression in testis. While we have used the set of 1,103 genes in the remaining analysis, one can identify the more restricted set of testis-specific genes in Supplementary Table 1.

\section{Identification of putative $\mathrm{CT}$ genes}

The expression pattern in tumors of the 1,103 genes predominantly expressed in testis was evaluated using the TCGA dataset. RNA-Seq data from 6,221 tumor samples were collected from the TCGA data repository comprising 15 tumor types. To identify genes significantly expressed in a given tumor, we used statistics provided by TCGA itself. A gene was considered a putative CT gene if it had a level of expression (cutoff threshold of RSEM >1) in at least $10 \%$ of all informative samples for a given tumor. These two criteria identified 745 putative CT genes (201 as new CT genes by comparing to the CT Database and data from Wang et al. [5]) significantly expressed in at least one tumor type (Figure 2A). If a more stringent threshold is used (15\% of all samples for a given tumor type), we found 678 putative CT genes (176 as new CT genes) (Figure 2A). The same procedure was 
applied to the list of testis-biased genes identified using the more stringent 0.99 proportional score giving 478 (155 new) and 418 (132 new) putative CT genes using the $10 \%$ and $15 \%$ thresholds, respectively (Figure 2A). Supplementary Table 2 lists all genes with their corresponding expression in all analyzed tumor types. A comparative analysis between all tumor types, shown in Figure $2 \mathrm{~B}$, reveals that among the putative $\mathrm{CT}$ genes one can find several that are tumor-specific. In several cases, a particular CT gene showed a significant expression in only one tumor (17\% of all CT genes). For example, 32 CT genes were expressed exclusively in leukemia, 11 in melanoma, and 14 in ovarian cancer (Figure 2B). This finding demonstrated the potential of our strategy for proposing particular biomarkers and targets for different types of tumors.

\section{CT genes as cancer genes?}

Much has been discussed on the role of CT genes in driving tumorigenesis [12]. Based on that, we decided to explore our set of putative CT genes regarding their status as cancer genes in the TCGA dataset used here. A method recently developed by us, the S-score [21], integrates information such as mutation screening, methylation status, copy-number variation and expression profiling and was used to infer whether our CT candidates showed a pattern of either an oncogene or tumor suppressor. The S-score was calculated for all 1,103 genes preferentially expressed in testis for all 15 tumor types studied here. When a stringent cut-off was used, as in De Souza et al. [21] (S-score $>3$, indicating an oncogene, or $<-3$, showing a tumor suppressor), we found 313 cancer genes (128

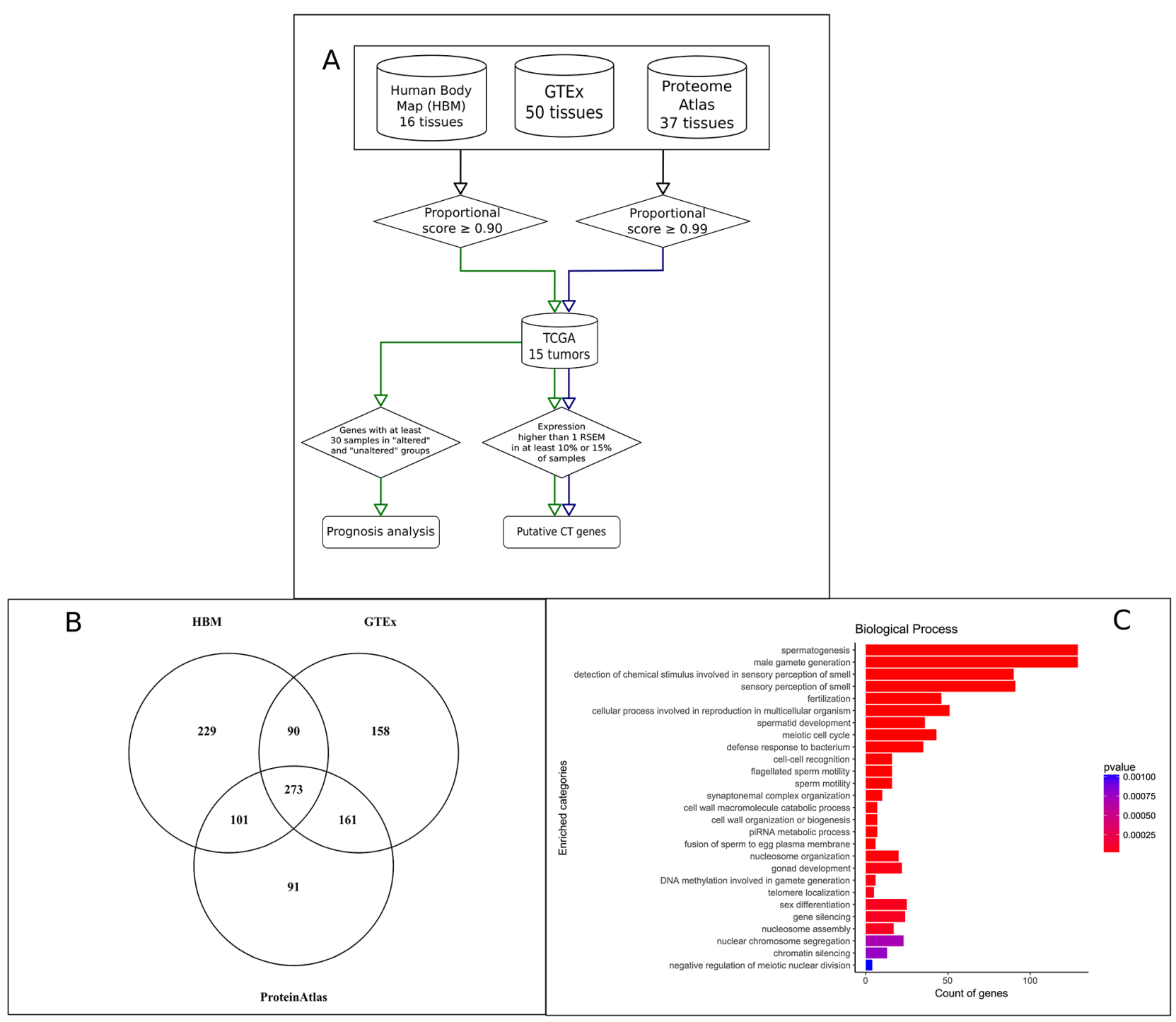

Figure 1: Identification of putative CT genes in 15 tumor types. (A) Schematic view of the pipeline used to identify CT genes with the identification of 1,103 genes predominant or exclusively expressed in testis and 745 putative CT genes. (B) Venn diagram showing the intersection of the 1103 genes predominant or exclusively expressed in testis regarding their source. (C) Gene Ontology enrichment analysis using the set of 1,103 genes predominant or exclusively expressed in testis. 
oncogenes and 201 tumor suppressors) among the 1,103 genes preferentially expressed in testis. Few genes behave differently in distinct tumor types. Supplementary Table 2 lists the S-score values for all testis-biased genes in all 15 tumor types used here.

To evaluate whether the set of putative CT genes is enriched or depleted of cancer genes in any tumor type, a Monte Carlo simulation was performed using random sets of 1,103 genes. As shown in Figure 3, there is an overall depletion of oncogenes in this set of testis-biased genes (pattern found in 12 out of 15 tumor types). Only five tumor types showed a depletion of suppressors while two tumor types showed an enrichment of suppressors (LUAD and SKCM). No oncogene enrichment was found. The same pattern, general depletion of cancer genes in the set of CT genes, was also observed when we used the set of 745 putative CT genes in the simulations (Supplementary Figure 1). This finding strongly suggests that, while there are several cancer genes within the CT genes dataset, there is overall a depletion of cancer genes in that set except for SKCM and LUAD, both presenting enrichment for tumor suppressors.

A

\begin{tabular}{|c|c|c|}
\hline \multirow{2}{*}{$\begin{array}{c}\text { Proportional score } \\
\text { (\# genes) }\end{array}$} & \multicolumn{2}{|c|}{ Number of putative CTs } \\
\cline { 2 - 3 } & $10 \%$ (\# new-CTs) & $15 \%$ (\# new-CTs) \\
\hline $90 \%(1103)$ & $745(201)$ & $678(176)$ \\
\hline $99 \%(793)$ & $478(155)$ & $418(132)$ \\
\hline
\end{tabular}

B

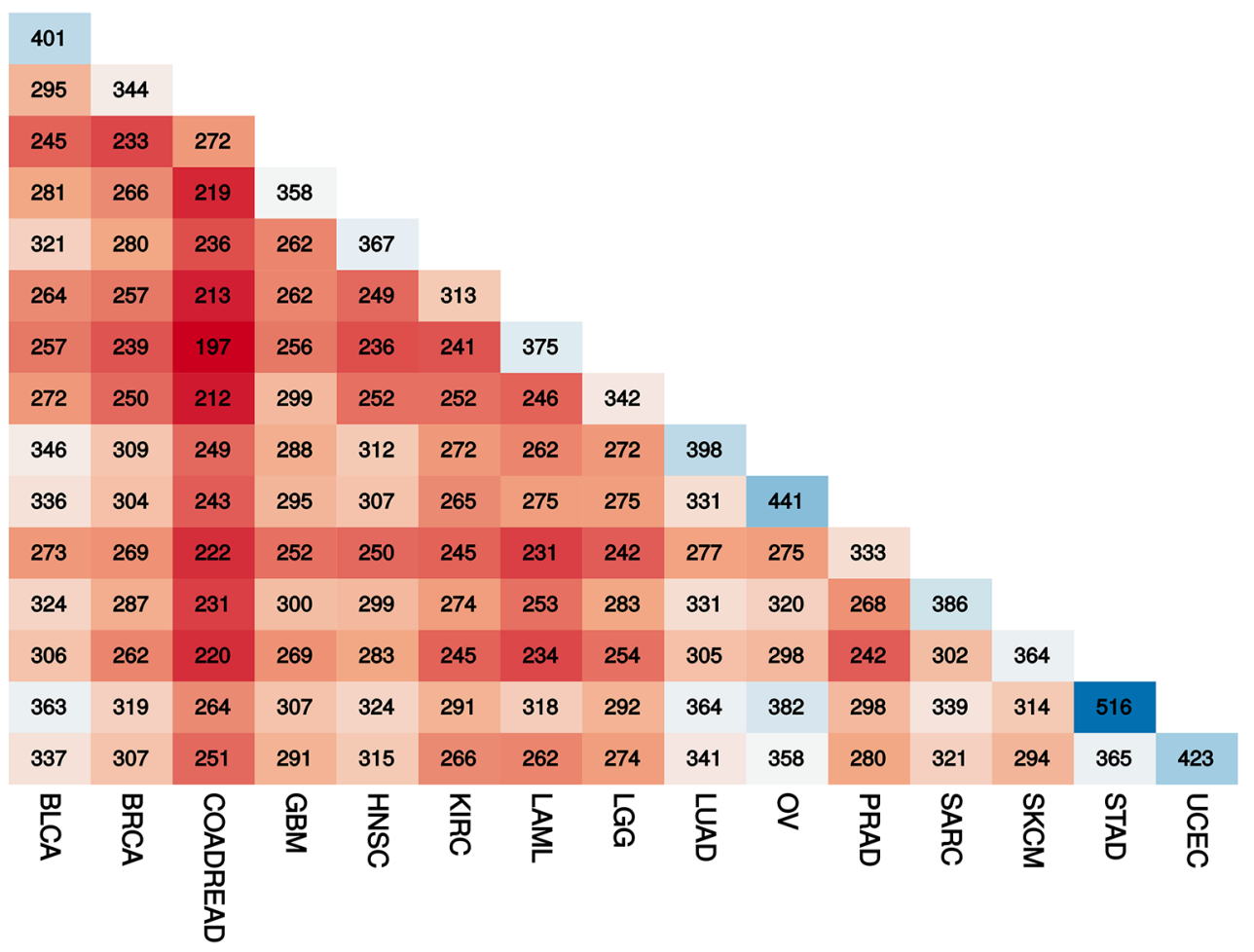

Tumor Specific CTs

2 - BLCA

2 - BRCA

3 - COADREAD

3 - GBM

5 - HNSC

4 - KIRC

32 - LAML

9 - LGG

2 - LUAD

14 - OV

10 - PRAD

2 - SARC

11 - SKCM

29 - STAD

3 - UCEC

Figure 2: Comparative analysis of putative CT genes. (A) Number of testis-biased genes and putative CT genes according to different stringent criteria for selection. (B) Matrix reporting the number of shared CT genes for all possible paired tumor type. The list at the right is the number of exclusive CT genes per tumor. 


\section{Protein products of CT genes detected in tumor samples}

To serve either as a biomarker or a therapeutic target, a given CT gene should be expressed at the protein level. Mass spectrometry-based strategies are becoming powerful resources to query the proteome, and the golden standards technologies are now able to identify around 10,000 proteins per sample. We capitalize on the availability of mass spectrometry data from different sources for a variety of tumor samples to determine among the putative CT genes those expressed at the protein level.

Using a cohort of 209 samples from different tumor types (04 melanoma, 95 colorectal tumors, 40 breast tumors, 36 prostate tumors and 34 ovary tumors), we identified 136 putative CT genes at the protein level (Supplementary Table 3). Although this is a significant number, it should be emphasized that a high rate of false-negatives is expected in this analysis due to the non-exhaustive nature of mass spectrometry-based approaches. By plotting the sum of the area under curve measurements of all identified proteins in each sample group (Supplementary Figure 2), it is possible to observe that many of these CT gene products belong to the top
$50 \%$ of the most abundant proteins in the detectable proteome of the sample.

A comparison of the Top 15 most abundant CT genes in the proteomic dataset shows that some genes are more globally present in most or all tumors represented here, such as PBK, SPATA22, IL4I1, HIST1H1A, among other. Some genes seem to show a more specific profile depending on the tumor type, such as C17ORF104, only highly-abundant in the melanoma samples, FUT5 in colon, PAGE1 in ovarian tumor and CSNK1A1L in prostate.

\section{CT signature for cancer prognosis}

Clinical data regarding overall survival from TCGA patients was used to evaluate the impact of expression of a putative $\mathrm{CT}$ gene in the outcome of the corresponding patients. A computer program evaluated all putative CT genes (745) for all 15 tumor types regarding any survival difference between samples expressing (FPKM or RSEM $>1$ ) or not expressing a given CT gene. Supplementary Table 4 provides the raw data for this survival analysis for all tumor types. Genes reporting a q-value $<0.05$ (as defined by a log-rank test) for the difference in survival between the groups of patients expressing or

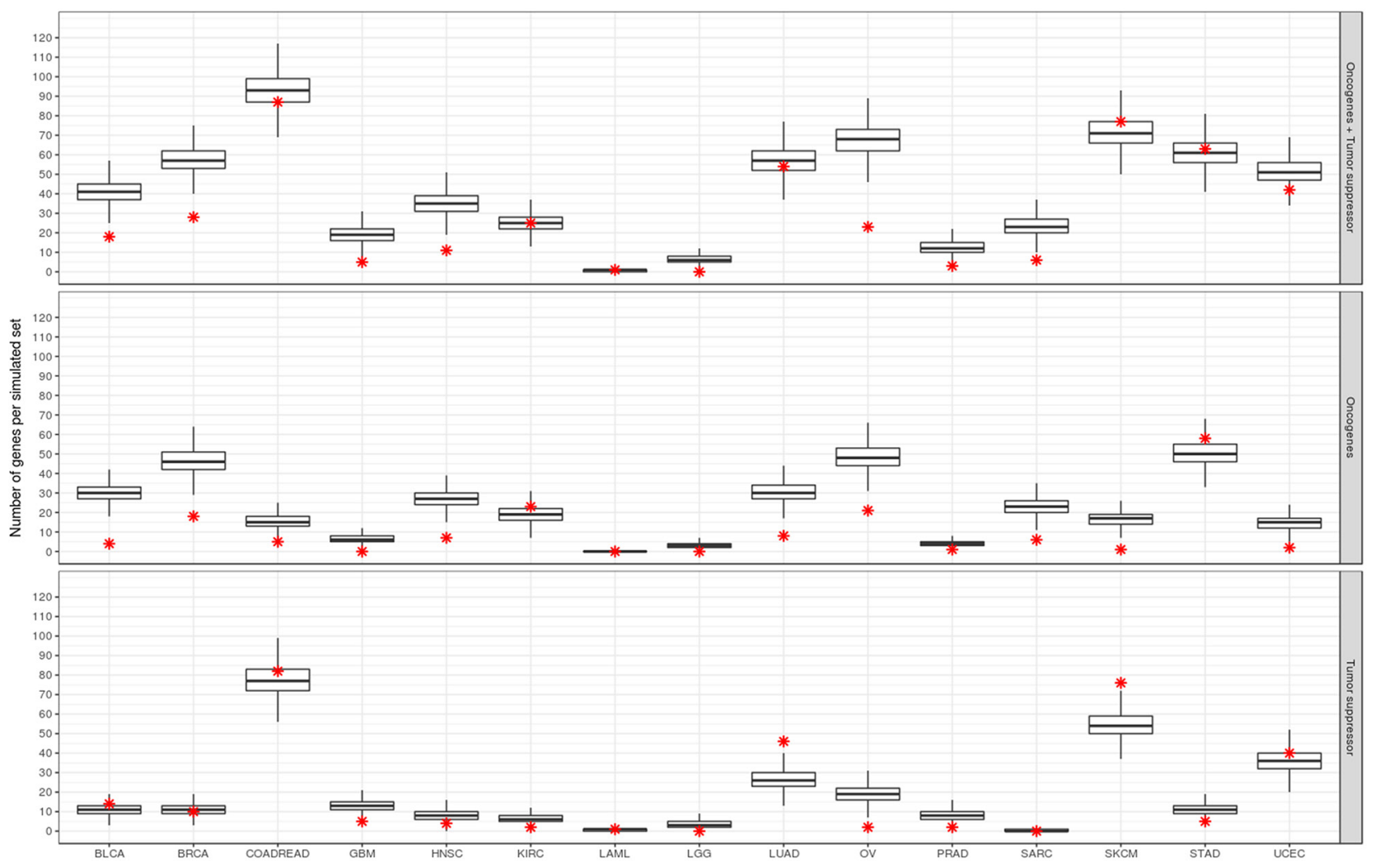

Figure 3: Enrichment analysis of CT genes for oncogenes and tumor suppressors. Box plots represent the distribution of the number of cancer genes in the 10,000 Monte Carlo simulations. Red star indicates the true number of cancer genes in the set of CT genes for each respective tumor type. Upper, middle and lower panels correspond to the real and simulated sets for oncogenes and suppressors together, oncogenes and suppressors, respectively. 
not expressing a given CT gene were selected for further analysis. Overall we found 207 CT genes (non-redundant) whose expression affected the outcome of patients in at least one tumor type. Expression of CT genes was more associated with poor prognosis (179 genes) than to better prognosis (57 genes). Few genes behave differently in distinct tumor types. We confirmed the finding from Yao et al. [20] who found a high frequency of CT genes affecting prognosis in kidney renal clear cell carcinoma (KIRC). Most of the CT genes in KIRC were associated with poor prognosis (129 out of 141 genes). A more even distribution of CT genes associated to good and poor prognosis were found for LGG (30 and $32 \mathrm{CT}$ genes, respectively) and SKCM (5 and 5 CT genes, respectively). To further select potential candidates, we split samples based on the expression of a given CT gene in three categories: no expression, expression below the median and expression above the median. If the expression of a corresponding CT gene was truly associated with patient outcome, we would expect that patients expressing more of the corresponding CT gene would have a stronger survival effect. A manual inspection of the Kaplan-Meyer plots for all $236 \mathrm{CT}$ genes (179 and 57 associated to poor and good prognosis, respectively) was performed looking for the above pattern, and 113 genes were found more strongly associated with patient outcome (illustrative plots for KIRC and SKCM are shown in Figure 4).

Data from Senbabaoglu et al. [22], who also used expression data from TCGA, was then used to assess the association between expression of a given $\mathrm{CT}$ gene and the number of infiltrating CD8+ cells. Senbabaoglu et al. [22] developed a method that uses expression data from TCGA to estimate the number of imune cells infiltration in a given tumor. Our analysis was only possible for four tumor types (BLCA, HNSC, LUAD and SKCM) due to either the lack of a significant number of CT genes associated with good prognosis or the lack of data of infiltrating CD8+ cells for some tumors. For two tumor types, LGG and KIRC, the high number of CT genes associated to both, good and poor prognosis, rendered the comparison impossible since both groups of samples were almost identical (since they comprised the totality of the samples). Figure 5A shows that samples with high expression of CT genes associated with good prognosis have a significantly higher number of CD8+ cells in BLCA $\left(p<9.9 \mathrm{e}^{-5}\right)$, HNSC $\left(\mathrm{p}<9.5 \mathrm{e}^{-4}\right)$ and SKCM $\left(1.1 \mathrm{e}^{-6}\right)$. When we split the samples according to the expression of a given CT gene, for most of the genes associated with good prognosis there is a significant association between the CT gene expression and infiltration of CD8+ cells for SKCM (Figure 5B), HNSC (Figure 5C) and BLCA (Figure 5D). A scatchard plot (showing the association between expression and CD8+ infiltration) for the same genes is shown in Supplementary Figure 3. Stronger associations were obtained for ZNF683 in BLCA, GPR31 in HNSC and C5ORF58, GTSF1L, HSF5 and HEATR9 in SKCM.

\section{DISCUSSION}

We have capitalized on the availability of NGS data to perform one the largest screening for $\mathrm{CT}$ genes so far. One first critical issue, as discussed by [5] and [14], is the classification of CT genes based on its expression pattern in normal tissues. These genes exhibit different expression profiles and can be categorized into testis-restricted, testis/ brain-restricted, or testis-selective group. This pattern imposes a challenge to identify the CT genes that are most suitable for the development of cancer therapies. Hofmann et al. [14], for example, performed an expression survey of 153 known CT genes and showed that only 39 had an exclusive expression in testis while the remaining had at least some expression in other tissues. Our screening of genes with a testis-biased expression involved the use of two proportional scores with different levels of stringency (0.9 and 0.99). While we used the less stringent dataset in most of the remaining analysis, the more stringent dataset is available to the community (Supplementary Table 1).

Several reports in the literature have indicated that CTs are mainly expressed in lung, ovarian, bladder, breast tumors and especially melanoma [3]. Most of the studies so far have used approaches based on the interrogation of few genes although Hofmann [14] used a series of highthroughput gene expression analyses to validate putative CT genes and Wang et al. [5] have used TCGA data from 19 tumor types. TCGA has provided a unique opportunity to screen for putative CT genes in a large panel of samples from many different tumor types and associated clinical features. Yao [20] have used TCGA data to explore the pan-cancer expression landscape of CT genes restricting their analysis to a subset of the CT database. Here, again, we used two thresholds with different levels of stringency (expression in at least $10 \%$ or $15 \%$ of samples in a given tumor) to identify putative CT genes. This allowed us to identify 745 putative CT genes (using the 10\% threshold). By comparing this set of CT genes with the catalogs from the CT database and from Wang et al. [5] we found 201 new CT genes in our dataset. Several CT genes showed expression in only one tumor, which demonstrate the potential of our analysis for proposing biomarkers and targets for particular types of tumor.

The CT gene catalog generated by this study allowed us to evaluate some questions regarding $\mathrm{CT}$ genes. For example, much has been discussed on the role of CT genes in driving tumorigenesis [12]. One of the arguments that support a more important role of CT genes in tumorigenesis is related to their functions, many of which are related to tumorigeneses, like signal transduction and gene regulation. Another indirect support comes from an apparent similarity between germ cell and tumor developments $[12,23,24]$. In that line, some authors $[25,26]$ proposed that the expression of CT genes, usually restricted to germline cells, would trigger a gametogenic program in other somatic cells that would 
contribute to the tumorigenesis. Furthermore, some CT genes seem to be associated with the maintenance of an undifferentiated state of stem cells as reported by Cheng et al. [27] and Lifantsenva et al. [28]. Nevertheless, few
CT genes have been shown to act as an oncogene (which would be expected based on their expression pattern). CT45A1 was shown to work as an oncogene and drive tumorigenesis in breast cancer [29]. Deletion of SSX2 in

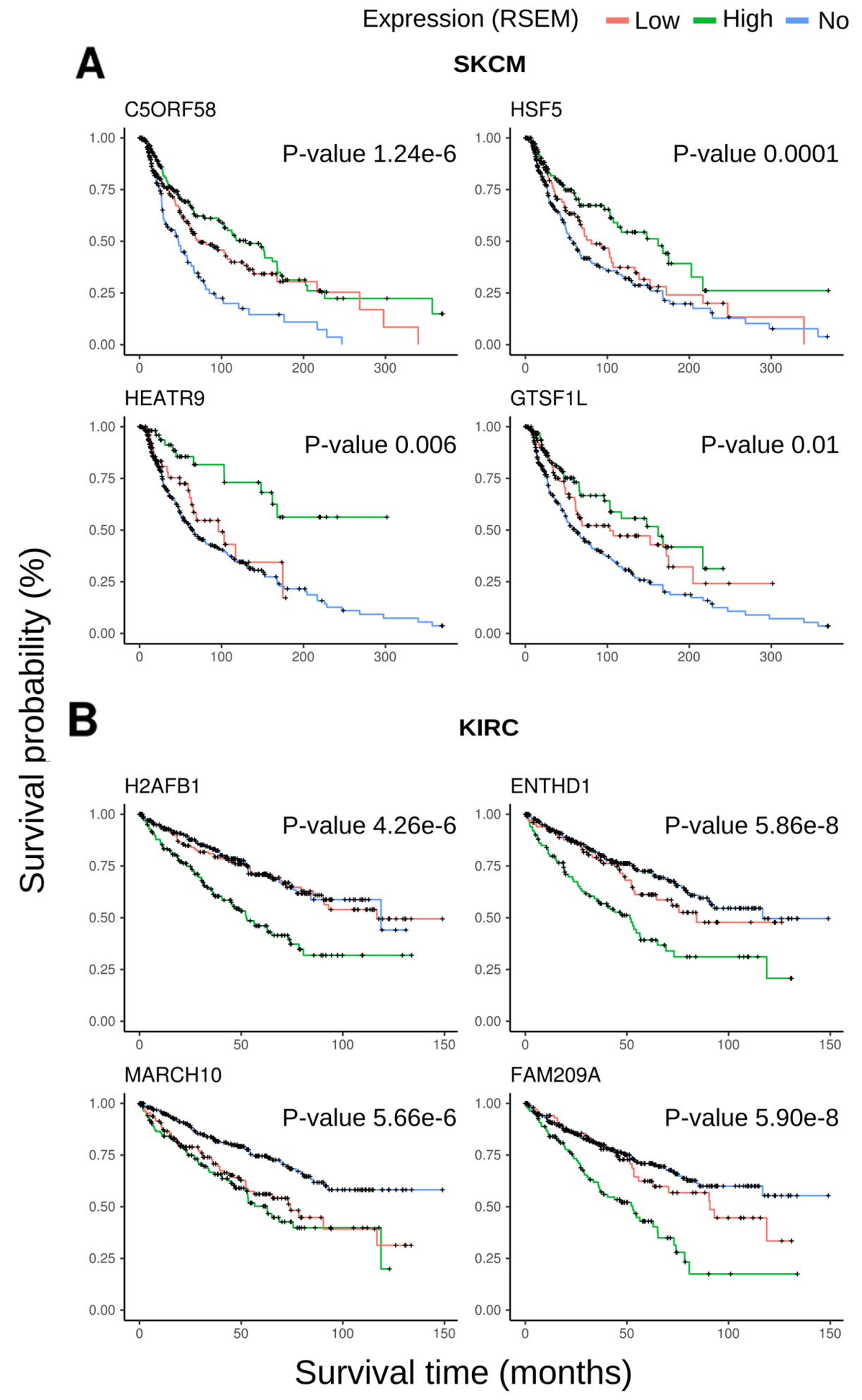

Figure 4: Kaplan-Meyer plots for representative CT genes. (A) Most representative plots for SKCM including four genes associated to good prognosis. (B) Most representative plots for KIRC including four genes associated to poor prognosis. 
melanoma cells significantly reduced cell proliferation [30]. Members of the MAGE family have been implicated in cancer cell survival, frequently acting in the p53 pathway $[31,32]$. Involvement with cancer progression, especially metastasis formation, has also been shown for some CT genes [29, 31, 33]. Our list of CT genes was evaluated for any potential enrichment of oncogenes and tumor suppressor, using the S-score method developed
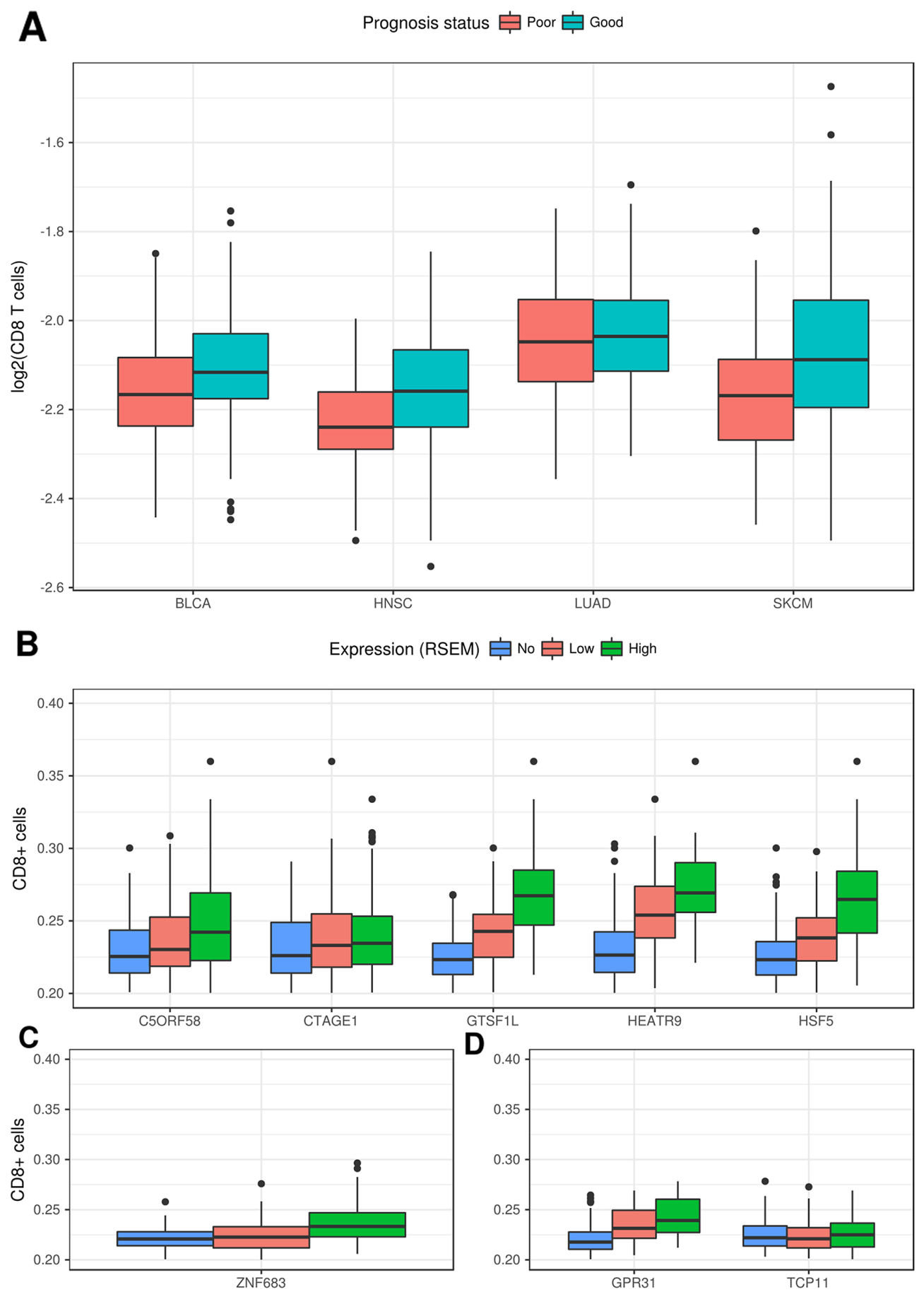

D

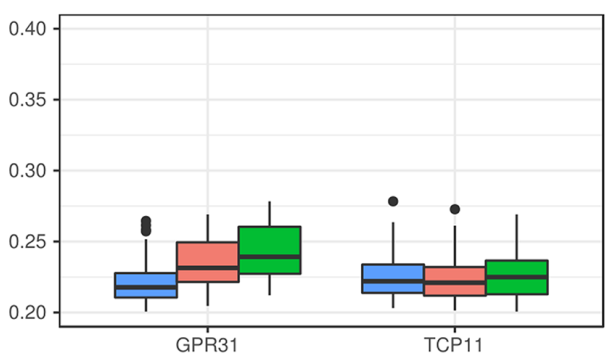

Figure 5: Correlation between expression of CT genes and the number of infiltrating CD8+ cells. (A) Samples with higher expression for CT genes associated to good prognosis have a higher rate of infiltrating CD8+ cells for BLCA, HNSC and SKCM. (B) Rate of infiltrating CD8+ cells for samples with no, low or high expression for the respective CT genes associated to good prognosis in SKCM. (C) Rate of infiltrating CD8+ cells for samples with no, low or high expression for the respective CT genes associated to good prognosis in HNSC. (D) Rate of infiltrating CD8+ cells for samples with no, low or high expression for the respective CT gene associated to good prognosis in BLCA. 
by us previously [21]. We found that in general there is a depletion of cancer genes in the set of CT genes. This finding strongly suggests that, while there are several cancer genes within the $\mathrm{CT}$ genes dataset, there is overall a depletion of cancer genes in that set except for melanoma and lung adenocarcinoma, both presenting enrichment for tumor suppressors.

Although few CT genes have been associated with either poor or good prognosis in a variety of tumor types [34-37], no large-scale analysis has been performed with a complete set of putative CT genes. Djureinovic et al. [38] generated a wide list of putative $\mathrm{CT}$ genes in non-small-cell lung cancer and found no gene associated to prognosis. Yao et al. [20] have recently shown that although some CT genes from the CT Database are associated with patient outcome, not many are independent prognostic markers. We explore this issue in a systematic way through an exhaustive analysis on the association between the expression of $\mathrm{CT}$ genes and cancer prognosis. Dozens of CT genes were associated with either good or poor prognosis. The robustness of our method is shown by the identification of genes clearly associated with disease progression among the set of $113 \mathrm{CT}$ genes more strongly related to patient outcome by our analysis. Included in this set of genes are TEX101 [39], HORMAD2 [40], OTP [41] and TEX19 [42]. It is interesting to notice that CT genes are not significantly enriched for genes associated to prognosis (data not shown).

It is tempting to speculate that the CT genes associated to a better prognosis are eliciting an immune response against the tumor, which could be the reason for a better outcome in such patients. ROPN1 has been demonstrated to induce autoantibodies in patients with prostate cancer [43] and multiple myeloma [44]. Spontaneous tumor immune response was also detected for SPAG6 in sera from patients with gastric cancer, melanoma and prostate cancer [45]. CTAGE1 antibodies were also found in sera of colorectal cancer patients [46].

The finding that expression of several CT genes is associated with good prognosis led us to hypothesize that this effect could be the result of infiltrating CD8+ cells driven by the CT gene expression. Several methods have been developed that evaluate the intra-tumor immune landscape based on gene expression analysis of the bulk tumor [22, 47, 48]. Data from Senbabaoglu et al. [22] is quite suitable for our analysis since they used their method in most of the tumor types evaluated by us in this report. We found several CT genes, especially in SKCM, whose expression is significantly associated to both good prognosis and CD8+ cell infiltration. We suggest that these $\mathrm{CT}$ genes be considered for further studies that would evaluate their immunotherapeutic potential.

\section{MATERIALS AND METHODS}

\section{RNA-Seq data source}

Four datasets were used for the identification of CT genes, including three from normal tissues, obtained from the Expression Atlas Portal [18]: the Human Body Map (GEO accession: GSE30611), GTEx [18] and Human Protein Atlas (HPA) [19]. The 16 samples from the Human Body Map were processed using our pipeline [49]. Expression values for GTEx and HPA datasets were obtained directly from the projects web page as FPKM and TPM values, respectively. Data from 15 tumor types from the TCGA consortium was used for the identification of putative CT genes. Expression, methylation and GISTIC $\mathrm{CNV}$ data were obtained from the cBIO portal by using the CGDS-R package, which provides processed data for each tumor type. Furthermore, somatic mutation data from COSMIC [50] and a local compilation of all somatic mutations found in the literature $[49,50]$ were used. For each sample in each tumor type, an expression threshold equal to 1 RSEM was applied to separate samples based on the expression of the putative CTs. Only genes with expression in more than $10 \%$ or $15 \%$ of samples were considered CTs for a particular tumor.

\section{Enrichment ontology analysis}

The R package "clusterProfiler" version 3.3 [51] was used to perform the ontology enrichment analysis based on Gene Ontology (GO) with a hyper-geometric test and correction method of Benjamini-Hochberg $(\mathrm{BH})$, with cutoff parameters of p-value $<0.05$ and q-value $<0.05$. To remove redundancy of enriched $\mathrm{GO}$ terms the function "simplify" with default parameters was used.

\section{Proteomic analysis of public cancer datasets}

The following MS raw file datasets were downloaded from ProteomeXchange: ovarian cancer dataset PXD003668 [52]; breast cancer dataset PXD002619 [53]; melanoma dataset PXD001724 [54]; colon cancer dataset PXD002041-50 [55]; prostate cancer dataset (PXD003430, PXD003452, PXD003515, PXD004132, PXD003615, PXD003636, PXD004159) [56]. All datasets were submitted to MaxQuant software version 1.5.2.8 [57] for protein identification. Parameters were set as follows: protein $\mathrm{N}$-acetylation and methionine oxidation as variable modifications; carbamidomethylation of cysteine as fixed modification; first search error window of $20 \mathrm{ppm}$ and main search error of $6 \mathrm{ppm}$ at MS level. Furthermore, trypsin without proline restriction enzyme option was used, with two allowed mis-cleavages. Minimal unique peptides were set to 1, and FDR allowed was $0.01(1 \%)$ for peptide and protein identification. The 
Uniprot human database was used (download from August 2016). Generation of reversed sequences was selected to assign FDR rates. A contaminants filter was performed, removing all occurrences presents on columns "Reverse" or "Potential contaminant" from the output of MaxQuant.

\section{S-score simulation}

Identification of cancer genes was performed using the S-score metric [21] in both the set of testis-enriched genes (1103) and the set of 745 putative CTs. The Monte Carlo simulation was performed against each tumor type (with extreme S-score), where 10.000 simulated sets were compared to the real sets. In this step, three different tests were carried out: enrichment for oncogenes (genes with S-score $\geq 3$ ), enrichment for tumor suppressor (genes with S-score $\leq-3$ ) and enrichment for cancer genes (including both oncogenes and tumor suppressors).

\section{Survival signatures and patients prognosis}

To test the association of $\mathrm{CT}$ genes with patient outcome in a given tumor type all putative CTs expressed in at least 30 samples were used. All putative CTs were tested individually using a log-rank test and genes were selected based on a threshold (q-value $\leq 0.05$ ), as defined by the "qvalue" R package [58], and classified as associated with "Good" or "Poor" prognosis. Next, samples expressing a given $\mathrm{CT}$ associated with prognosis were separated in two subsets based on a median expression of the corresponding CT gene. KaplanMeyer curves were plotted using the ggplot2 (from the $\mathrm{R}$ package). CD8+ profiling for TCGA samples was obtained from Senbabaoglu et al. [22].

\section{Abbreviations}

CT: Cancer/testis; CG: cancer-germline; NGS: next-generation sequencing; TCGA: The Cancer Genome Atlas; HBM: Human Body Map; GTEx: The Genotype-Tissue Expression; KIRC: kidney renal clear cell carcinoma; SKCM: skin cutaneous melanoma; GO: Gene Ontology.

\section{ACKNOWLEDGMENTS AND FUNDING}

This work was supported by a CAPES grant (23038.004629/2014-19) to SJS, by the Ludwig Institute for Cancer Research to SJS, and by the Institute of Bioinformatics and Biotechnology to SJS. Data analyses were performed on supercomputers from the Digital Metropolis Institute at the Federal University of Rio Grande do Norte.

\section{CONFLICTS OF INTEREST}

The authors declare that they have no conflicts of interest.

\section{REFERENCES}

1. Krishnadas DK, Shusterman S, Bai F, Diller L, Sullivan JE, Cheerva AC, George RE, Lucas KG. A phase I trial combining decitabine/dendritic cell vaccine targeting MAGE-A1, MAGE-A3 and NY-ESO-1 for children with relapsed or therapy-refractory neuroblastoma and sarcoma. Cancer Immunol Immunother. 2015; 64:1251-60.

2. Scanlan MJ, Simpson AJG, Old LJ. The cancer/testis genes: Review, standardization, and commentary. Cancer Immun. 2004; 4:1-15.

3. Simpson AJG, Caballero OL, Jungbluth A, Chen YT, Old LJ. Cancer/testis antigens, gametogenesis and cancer. Nat Rev Cancer. 2005; 5:615-25.

4. Koslowski M, Bell C, Seitz G, Lehr H, Roemer K, Mu H. Frequent Nonrandom Activation of Germ-Line Genes in Human Cancer. Cancer Res. 2004; 64:5988-93.

5. Wang C, Gu Y, Zhang K, Xie K, Zhu M, Dai N, Jiang Y, Guo X, Liu M, Dai J, Wu L, Jin G, Ma H, et al. Systematic identification of genes with a cancer-testis expression pattern in 19 cancer types. Nat Commun. 2016; 7:10499.

6. Akcakanat A, Kanda T, Koyama Y, Watanabe M, Kimura E, Yoshida Y, Komukai S, Nakagawa S, Odani S, Fujii H, Hatakeyama K. NY-ESO-1 expression and its serum immunoreactivity in esophageal cancer. Cancer Chemother Pharmacol. 2004; 54:95-100.

7. Gnjatic S, Atanackovic D, Jäger E, Matsuo M, Selvakumar A, Altorki NK, Maki RG, Dupont B, Ritter G, Chen YT, Knuth A, Old LJ. Survey of naturally occurring CD4+ T cell responses against NY-ESO-1 in cancer patients: correlation with antibody responses. Proc Natl Acad Sci U S A. 2003; 100:8862-7.

8. Ayyoub M, Zippelius A, Pittet MJ, Rimoldi D, Valmori D, Cerottini JC, Romero P, Lejeune F, Liénard D, Speiser DE. Activation of human melanoma reactive CD8+ T cells by vaccination with an immunogenic peptide analog derived from Melan-A/melanoma antigen recognized by T cells- 1 . Clin Cancer Res. 2003; 9:669-77.

9. Ayyoub M, Rimoldi D, Guillaume P, Romero P, Cerottini JC, Valmori D, Speiser D. Tumor-reactive, SSX-2-specific $\mathrm{CD} 8+\mathrm{T}$ cells are selectively expanded during immune responses to antigen-expressing tumors in melanoma patients. Cancer Res. 2003; 63:5601-6.

10. Ayyoub M, Brehm M, Metthez G, Talbot S, Dutoit V, Taub RN, Keohan ML, Gure AO, Chen YT, Williamson B, Jungbluth AA, Old LJ, Hesdorffer CS, et al. SSX antigens 
as tumor vaccine targets in human sarcoma. Cancer Immun. 2003; 3:1-8.

11. Tsuji T, Altorki NK, Ritter G, Old LJ, Gnjatic S. Characterization of Preexisting MAGE-A3-Specific CD4+ $\mathrm{T}$ Cells in Cancer Patients and Healthy Individuals and Their Activation by Protein Vaccination. J Immunol. 2009; 183:4800-8.

12. GjerstorffMF,Andersen MH, Ditzel HJ. Oncogenic cancer/testis antigens: prime candidates for immunotherapy. Oncotarget. 2015; 6:15772-87. https://doi.org/10.18632/oncotarget.4694.

13. Scanlan MJ, Gure AO, Jungbluth AA, Old LJ, Chen YT. Cancer/testis antigens: an expanding family of targets for cancer immunotherapy. Immunol Rev. 2002; 188:22-32.

14. Hofmann O, Caballero OL, Stevenson BJ, Chen YT, Cohen T, Chua R, Maher CA, Panji S, Schaefer U, Kruger A, Lehvaslaiho M, Carninci P, Hayashizaki Y, et al. Genomewide analysis of cancer/testis gene expression. Proc Natl Acad Sci U S A. 2008; 105:20422-7.

15. Almeida LG, Sakabe NJ, de Oliveira AR, Silva MCC, Mundstein AS, Cohen T, Chen YT, Chua R, Gurung S, Gnjatic S, Jungbluth AA, Caballero OL, Bairoch A, et al. CTdatabase: A knowledge-base of high-throughput and curated data on cancer-testis antigens. Nucleic Acids Res. 2009; 37:2007-10.

16. da Cunha JPC, Galante PAF, de Souza JE, de Souza RF, Carvalho PM, Ohara DT, Moura RP, Oba-Shinja SM, Marie SKN, Silva WA, Perez RO, Stransky B, Pieprzyk M, et al. Bioinformatics construction of the human cell surfaceome. Proc Natl Acad Sci. 2009; 106:16752-7.

17. Lee SY, Obata Y, Yoshida M, Stockert E, Williamson B, Jungbluth AA, Chen YT, Old LJ, Scanlan MJ. Immunomic analysis of human sarcoma. Proc Natl Acad Sci U S A. 2003; 100:2651-6.

18. Petryszak R, Keays M, Tang YA, Fonseca NA, Barrera E, Burdett T, Füllgrabe A, Fuentes AMP, Jupp S, Koskinen S, Mannion O, Huerta L, Megy K, et al. Expression Atlas update - An integrated database of gene and protein expression in humans, animals and plants. Nucleic Acids Res. 2016; 44:D746-52.

19. Uhlen M, Fagerberg L, Hallstrom BM, Lindskog C, Oksvold P, Mardinoglu A, Sivertsson A, Kampf C, Sjostedt E, Asplund A, Olsson I, Edlund K, Lundberg E, et al. Tissue-based map of the human proteome. Science. 2015; 347:1260419-1260419.

20. Yao J, Caballero OL, Yung WKA, Weinstein JN, Riggins GJ, Strausberg RL, Zhao Q. Tumor Subtype-Specific Cancer-Testis Antigens as Potential Biomarkers and Immunotherapeutic Targets for Cancers. Cancer Immunol Res. 2014; 2:371-9.

21. De Souza JES, Fonseca AF, Valieris R, Carraro DM, Wang JYJ, Kolodner RD, De Souza SJ. S-score: A scoring system for the identification and prioritization of predicted cancer genes. PLoS One. 2014; 9:1-9.

22. Şenbabaoğlu Y, Gejman RS, Winer AG, Liu M, Van Allen EM, de Velasco G, Miao D, Ostrovnaya I, Drill E, Luna A, Weinhold
N, Lee W, Manley BJ, et al. Tumor immune microenvironment characterization in clear cell renal cell carcinoma identifies prognostic and immunotherapeutically relevant messenger RNA signatures. Genome Biol. 2016; 17:231.

23. Caballero OL, Chen YT. Cancer/testis (CT) antigens: Potential targets for immunotherapy. Cancer Sci. 2009; 100:2014-21.

24. Rousseaux S, Debernardi A, Jacquiau B, Vitte AL, Vesin A, Nagy-Mignotte H, Moro-Sibilot D, Brichon PY, Lantuejoul S, Hainaut P, Laffaire J, de Reynies A, Beer DG, et al. Ectopic Activation of Germline and Placental Genes Identifies Aggressive Metastasis-Prone Lung Cancers. Sci Transl Med. 2013; 5:186ra66-186ra66.

25. Old LJ. Cancer is a somatic cell pregnancy. Cancer Immun. 2007; 7:19.

26. Akers SN, Odunsi K, Karpf AR. Regulation of cancer germline antigen gene expression: implications for cancer immunotherapy. Futur Oncol. 2010; 6:717-32.

27. Cheng YH, Wong EW, Cheng CY. Cancer/testis (CT) antigens, carcinogenesis and spermatogenesis. Spermatogenesis. 2011; 1:209-20.

28. Lifantseva N, Koltsova A, Krylova T, Yakovleva T, Poljanskaya G, Gordeeva O. Expression Patterns of CancerTestis Antigens in Human Embryonic Stem Cells and Their Cell Derivatives Indicate Lineage Tracks. Stem Cells Int. 2011; 2011:1-13.

29. Shang B, Gao A, Pan Y, Zhang G, Tu J, Zhou Y, Yang P, Cao Z, Wei Q, Ding Y, Zhang J, Zhao Y, Zhou Q. CT45A1 acts as a new proto-oncogene to trigger tumorigenesis and cancer metastasis. Cell Death Dis. 2014; 5:e1285.

30. Greve KB, Lindgreen JN, Terp MG, Pedersen CB, Schmidt S, Mollenhauer J, Kristensen SB, Andersen RS, Relster MM, Ditzel HJ, Gjerstorff MF. Ectopic expression of cancer/testis antigen SSX2 induces DNA damage and promotes genomic instability. Mol Oncol. 2015; 9:437-49.

31. Yang B, O'Herrin SM, Wu J, Reagan-Shaw S, Ma Y, Bhat KMR, Gravekamp C, Setaluri V, Peters N, Hoffmann FM, Peng H, Ivanov AV, Simpson AJG, et al. MAGE-A, mMage-b, and MAGE-C proteins form complexes with KAP1 and suppress p53-dependent apoptosis in MAGEpositive cell lines. Cancer Res. 2007; 67:9954-62.

32. Marcar L, MacLaine NJ, Hupp TR, Meek DW. Mage-A cancer/testis antigens inhibit p53 function by blocking its interaction with chromatin. Cancer Res. 2010; 70:10362-70.

33. Caballero OL, Shousha S, Zhao Q, Simpson AJG, Coombes RC, Neville AM. Expression of Cancer/Testis genes in ductal carcinoma in situ and benign lesions of the breast. Oncoscience. 2013; 1:14-20. https://doi.org/10.18632/ oncoscience.4.

34. Sang M, Gu L, Yin D, Liu F, Lian Y, Zhang X, Liu S, Huang $\mathrm{W}, \mathrm{Wu} \mathrm{Y}$, Shan B. MAGE-A family expression is correlated with poor survival of patients with lung adenocarcinoma: a retrospective clinical study based on tissue microarray. $\mathrm{J}$ Clin Pathol. 2016. 
35. Ohue Y, Kurose K, Nozawa R, Isobe M, Nishio Y, Tanaka T, Doki Y, Hori T, Fukuoka J, Oka M, Nakayama E. Survival of Lung Adenocarcinoma Patients Predicted from Expression of PD-L1, Galectin-9, and XAGE1 (GAGED2a) on Tumor Cells and Tumor-Infiltrating T Cells. Cancer Immunol Res. 2016; 4:1049-60.

36. Liu S, Sang M, Xu Y, Gu L, Liu F, Shan B. Expression of MAGE-A1, -A9, -A11 in laryngeal squamous cell carcinoma and their prognostic significance: a retrospective clinical study. Acta Otolaryngol. 2016; 136:506-13.

37. Ohue Y, Wada H, Oka M, Nakayama E. Antibody response to cancer/testis (CT) antigens: A prognostic marker in cancer patients. Oncoimmunology. 2014; 3:e970032.

38. Djureinovic D, Hallström BM, Horie M, Mattsson JSM, La Fleur L, Fagerberg L, Brunnström H, Lindskog C, Madjar K, Rahnenführer J, Ekman S, Ståhle E, Koyi H, et al. Profiling cancer testis antigens in non-small-cell lung cancer. JCI insight. 2016; 1:e86837.

39. Yoshitake H, Yokoi H, Ishikawa H, Maruyama M, Endo S, Nojima M, Yoshida K, Yoshikawa H, Suzuki F, Takamori K, Fujiwara H, Araki Y. Overexpression of TEX101, a potential novel cancer marker, in head and neck squamous cell carcinoma. Cancer Biomark. 2013; 12:141-8.

40. Zhang K, Tang S, Cao S, Hu L, Pan Y, Ma H, Guo X, $\mathrm{Wu} \mathrm{S}$, Shen $\mathrm{H}, \mathrm{Hu} \mathrm{Z}$. Association of polymorphisms at HORMAD2 and prognosis in advanced non-small-cell lung cancer patients. Cancer Epidemiol. 2014; 38:414-8.

41. Swarts DRA, Henfling MER, Van Neste L, Van Suylen RJ, Dingemans AMC, Dinjens WNM, Haesevoets A, Rudelius M, Thunnissen E, Volante M, Van Criekinge W, Van Engeland M, Ramaekers FCS, et al. CD44 and OTP are strong prognostic markers for pulmonary carcinoids. Clin Cancer Res. 2013; 19:2197-207.

42. Zhong J, Chen Y, Liao X, Li J, Wang H, Wu C, Zou X, Yang G, Shi J, Luo L, Liu L, Deng J, Tang A. Testis expressed 19 is a novel cancer-testis antigen expressed in bladder cancer. Tumor Biol. 2016; 37:7757-65.

43. Adeola HA, Smith M, Kaestner L, Blackburn JM, Zerbini LF. Novel potential serological prostate cancer biomarkers using CT100+ cancer antigen microarray platform in a multi-cultural South African cohort. Oncotarget. 2016; 7:13945-64. https://doi.org/10.18632/oncotarget.7359.

44. Chiriva-Internati M, Mirandola L, Yu Y, Jenkins MR, Gornati R, Bernardini G, Gioia M, Chiaramonte R, Cannon MJ, Kast $\mathrm{WM}$, Cobos E. Cancer testis antigen, ropporin, is a potential target for multiple myeloma immunotherapy. J Immunother. $2011 ; 34: 490-9$.

45. Siliņa K, Zayakin P, Kalniņa Z, Ivanova L, Meistere I, Endzeliņš E, Ābols A, Stengrēvics A, Leja M, Ducena K, Kozirovskis V, Linē A. Sperm-associated Antigens as Targets for Cancer Immunotherapy. J Immunother. 2011; 34:28-44.

46. Gerhardt A, Usener D, Keese M, Sturm J, Schadendorf D, Eichmüller S. Tissue expression and sero-reactivity of tumor-specific antigens in colorectal cancer. Cancer Lett. 2004; 208:197-206.

47. Newman AM, Liu CL, Green MR, Gentles AJ, Feng W, Xu Y, Hoang CD, Diehn M, Alizadeh AA. Robust enumeration of cell subsets from tissue expression profiles. Nat Methods. 2015; 12:1-10.

48. Gong L, Parikh S, Rosenthal PJ, Greenhouse B. Biochemical and immunological mechanisms by which sickle cell trait protects against malaria. Malar J. 2013; 12:317.

49. Fonseca AL, da Silva VL, da Fonsêca MM, Meira ITJ, da Silva TE, Kroll JE, Ribeiro-dos-Santos AM, Freitas CR, Furtado R, de Souza JE, Stransky B, de Souza SJ. Bioinformatics Analysis of the Human Surfaceome Reveals New Targets for a Variety of Tumor Types. Int J Genomics. 2016; 2016:1-7.

50. Forbes SA, Beare D, Boutselakis H, Bamford S, Bindal N, Tate J, Cole CG, Ward S, Dawson E, Ponting L, Stefancsik R, Harsha B, Kok CY, et al. COSMIC: Somatic cancer genetics at high-resolution. Nucleic Acids Res. 2017; 45:D777-83.

51. Yu G, Wang LG, Han Y, He QY. clusterProfiler: an R Package for Comparing Biological Themes Among Gene Clusters. Omi A J Integr Biol. 2012; 16:284-7.

52. Coscia F, Watters KM, Curtis M, Eckert MA, Chiang CY, Tyanova S, Montag A, Lastra RR, Lengyel E, Mann M. Integrative proteomic profiling of ovarian cancer cell lines reveals precursor cell associated proteins and functional status. Nat Commun. 2016; 7:12645.

53. Tyanova S, Albrechtsen R, Kronqvist P, Cox J, Mann M, Geiger T. Proteomic maps of breast cancer subtypes. Nat Commun. 2016; 7:10259.

54. Welinder C, Pawłowski K, Sugihara Y, Yakovleva M, Jönsson G, Ingvar C, Lundgren L, Baldetorp B, Olsson H, Rezeli M, Jansson B, Laurell T, Fehniger T, et al. A protein deep sequencing evaluation of metastatic melanoma tissues. PLoS One. 2015; 10:1-16.

55. Zhang B, Wang J, Wang X, Zhu J, Liu Q, Shi Z, Chambers MC, Zimmerman LJ, Shaddox KF, Kim S, Davies SR, Wang S, Wang P, et al. Proteogenomic characterization of human colon and rectal cancer. Nature. 2014; 513:382-7.

56. Iglesias-Gato D, Wikström $\mathrm{P}$, Tyanova $\mathrm{S}$, Lavallee $\mathrm{C}$, Thysell E, Carlsson J, Hägglöf C, Cox J, Andrén O, Stattin P, Egevad L, Widmark A, Bjartell A, et al. The Proteome of Primary Prostate Cancer. Eur Urol. 2016; 69:942-52.

57. Cox J, Mann M. MaxQuant enables high peptide identification rates, individualized p.p.b.-range mass accuracies and proteome-wide protein quantification. Nat Biotechnol. 2008; 26:1367-72.

58. Storey JD, Bass AJ, Dabney A, Robinson D. qvalue: Q-value estimation for false discovery rate control. $\mathrm{R}$ package version 2.6.0. Bioconductor; 2015. Available from http://github.com/jdstorey/qvalue. 